\title{
THE FRACTIONAL FOURIER TRANSFORM AND THE WIGNER DISTRIBUTION
}

\author{
DAVID MUSTARD ${ }^{1}$
}

(Received 6 July 1994; revised 18 January 1995)

\begin{abstract}
The Wigner distribution and many other members of the Cohen class of generalized phasespace distributions of a signal all share certain translation properties and the property that their two marginal distributions of energy density along the time and along the frequency axes equal the signal power and the spectral energy density. A natural generalization of this last property is shown to be a certain relationship through the Radon transform between the distribution and the signal's fractional Fourier transform. It is shown that the Wigner distribution is now distinguished by being the only member of the Cohen class that has this generalized property as well as a generalized translation property. The inversion theorem for the Wigner distribution is then extended to yield the fractional Fourier transforms.
\end{abstract}

\section{Introduction}

The integer powers of the symmetrically defined Fourier-Plancherel operator $F$ on the Hilbert space $L^{2}\left(\mathbb{R}^{d}\right)$ form a cyclic group $\left\{F^{k}\right\}_{k \in \mathbb{Z}_{4}}$ of order 4 [7] and so it is natural to imbed that discrete group of operators into a continuous one. For $d=1$ E. U. Condon [5] in 1937 derived a one-parameter continuous set of integral operators $\left\{F_{\theta}\right\}_{\theta \in \mathbb{U}}$ (where $\mathbb{J}=\mathbb{R} / 2 \pi \mathbb{Z}$ ) that had the properties of unitarity, the group property, continuity in the group space and of imbedding $\left\{F^{k}\right\}_{k \in \mathbb{Z}_{4}}$. For $d \geq 1 \mathrm{~V}$. Bargmann [1] in 1961 found a corresponding version in his study of the unitary isomorphism between $L^{2}\left(\mathbb{R}^{d}\right)$ and V. Fock's [10] space of entire analytic functions of $d$ complex variables. M. Taylor's recent work on pseudo-differential operators [22] involves the same Lie group of integral operators, the "fractional" Fourier operators. I have shown $[16,17]$ constructions that even for the one-dimensional case led to an infinite number of distinct Lie group imbeddings.

Besides the intrinsic interest in a continuous imbedding there are important areas of application in quantum mechanics, signal analysis and physical optics. The work of

'School of Mathematics, University of New South Wales, Sydney, 2052 Australia

(C) Australian Mathematical Society, 1996, Serial-fee code 0334-2700/96 
Condon, Bargmann and Fock arose in the context of quantum mechanics. Also arising in this context was the work of E. P. Wigner [24] and L. Cohen [4] in constructing phase-space distribution functions, functions of conjugate variables (for example, position and momentum) that had the property that their integral with respect to one variable gave the correct quantum-mechanical marginal probability distribution with respect to the conjugate variable. This work of Wigner and Cohen has been widely discussed in the context of signal analysis (for example, $[3,2,9]$ ) where time and frequency are the conjugate variables, the distributions are "time-frequency distributions" and the marginal distributions for a signal $f(t)$ are its power $|f(t)|^{2}$ and its spectral energy density $|F f(\omega)|^{2}$. The recent study of G.B. Folland [11] considers phase-space distributions in the context of group representations, in particular of the Heisenberg and symplectic groups, that are significant for signal analysis and quantum mechanics.

Time-frequency distributions are relevant to the analysis of statistically non-stationary signals, speech or music, for example, in which the useful representations of the signal need to capture something of both its temporal and spectral apsects. The fractional Fourier transform has the same relevance since it allows signal representations along axes "between" time and frequency.

I have shown elsewhere $[15,17]$ a result relevant to quantum mechanics and communication theory: that requiring invariance under the group of fractional transforms leads to a new family of uncertainty principles, the first one of which is stronger than Heisenberg's. The invariant measure of overall spread described there is related to an invariant of the moment of inertia tensor of the Wigner distribution.

I am grateful to one of the referees for pointing out that this work also has applications to some current research in physical optics, the propagation of light in quadratic graded index media (see, for example, that by Ozaktas and others in [18] and the further references there).

In Section 2 I give a brief summary of a construction of the fractional Fourier transform and some of its elementary properties. (See [11], for example, for a thorough account.)

The main part of the paper, Section 3, then analyses the relationship between the fractional Fourier transform and the Cohen class of time-frequency distributions. It is well known that among the Cohen class there are many, including the Wigner distribution, that all have certain desirable properties relating to translations and marginal distributions along the two time and frequency axes. This paper shows that the natural generalizations of these properties to axes in all directions involves the fractional Fourier transform and that the natural generalization of the marginal distribution property to all directions requires that the time-frequency distribution be related to the fractional Fourier transform by the Radon transform. (The Radon transform is the transform that has recently received renewed attention, for example 
$[6,12,19,21]$, due to its role in the development of computer-aided tomography.) The principal result of this paper is the theorem that the Wigner distribution possesses these more stringent properties that are the natural generalizations of the familiar ones and, moreover, is the only distribution that does so. The standard inversion theorem for the Wigner distribution, which involves integration in the Wigner-distribution plane along lines of a special direction, is then extended to cover all directions, from which it yields the fractional Fourier transform.

The substance of this study was outlined in [14]. See [15] for some related work.

\section{The fractional Fourier transform}

The Fourier operator $F$ on $L^{1}(\mathbb{R}) \cap L^{2}(\mathbb{R})$ (extending to the Fourier-Plancherel operator on $L^{2}(\mathbb{R})$ ) can be defined symmetrically by

$$
F f(t)=\langle\exp (\mathrm{i} s t), f(s)\rangle
$$

where inner product and associated 2-norm are defined

$$
\langle f, g\rangle=(2 \pi)^{-1 / 2} \int_{\mathbb{R}} \bar{f}(x) g(x) d x \text { and }\|f\|=\langle f, f\rangle^{1 / 2} .
$$

One construction $[1,11,16,17]$ of the fractional Fourier operator $F_{\theta}(\theta \in \mathbb{T})$ is based on the fact [7] that the set of normalized Hermite functions $\left\{h_{n}\right\}_{n \in \mathbb{N}}$ defined by

$$
h_{n}(t)=\left(2^{n-1 / 2} n !\right)^{-1 / 2} \exp \left(-t^{2} / 2\right) H_{n}(t)
$$

(where $H_{n}(t)=\exp \left(t^{2}\right)(-d / d t)^{n} \exp \left(-t^{2}\right)$, the $n$th Hermite polynomial) is a complete orthonormal set of eigenfunctions under (2) for $F$ satisfying

$$
F h_{n}=e^{-\mathrm{i} n \pi / 2} h_{n}
$$

so that if $f$ has the Fourier-Hermite series

$$
f=\sum_{n \in \mathbb{N}}\left\langle h_{n}, f\right\rangle h_{n}
$$

its Fourier transform is

$$
F f=\sum_{n \in \mathbb{N}}\left\langle h_{n}, f\right\rangle e^{-\mathrm{i} n \pi / 2} h_{n}
$$

and so a "fractional" Fourier transform is naturally defined

$$
F^{\alpha} f=\sum_{n \in \mathbb{N}}\left\langle h_{n}, f\right\rangle e^{-\mathrm{i} n \alpha \pi / 2} h_{n} \quad(\alpha \in \mathbb{R})
$$


that is, writing $F^{\alpha}=F_{\theta}$ where $\theta=\alpha \pi / 2(\theta \in \mathbb{T})$,

$$
F_{\theta} f=\sum_{n \in \mathbb{N}}\left\langle h_{n}, f\right\rangle e^{-\mathrm{i} n \theta} h_{n} .
$$

Interchanging the order of integration and summation in (6) (provided $\theta / \pi \notin \mathbb{Z}$ ) one gets for $0<|\theta|<\pi$

$$
F_{\theta} f(t)=\left\langle K_{\theta}(s, t), f(s)\right\rangle \quad \text { where } \quad K_{\theta}(s, t)=\sum_{n \in \mathbb{N}} e^{\mathrm{in} \theta} h_{n}(s) \bar{h}_{n}(t) .
$$

The series for the kernel $K_{\theta}(s, t)$ of the integral operator in (7) can be evaluated in closed form $[1,11,16]$ yielding eventually the definition of $F_{\theta}$ for $0<|\theta|<\pi$ as

$$
F_{\theta} f(t)=\frac{C(\theta)}{\sqrt{2 \pi}} \int_{\mathbb{R}} \exp \left[-\mathrm{i} \frac{-\left(s^{2}+t^{2}\right) \cos \theta+2 s t}{2 \sin \theta}\right] f(s) d s
$$

where

$$
C(\theta)=|\sin \theta|^{-1 / 2} \exp \left[-\frac{\mathrm{i}}{2}\left(\frac{\pi}{2} \operatorname{sgn} \theta-\theta\right)\right]
$$

while for $\theta=0$ and $\pi$ one has $F_{0}=I$, the identity operator, and $F_{\pi}=F^{2}$, the parity operator.

Defining operators $D$ and $X$ by $(D f)(t)=(d / d t) f(t)$ and $(X f)(t)=t f(t)$ and then differential operators $J^{+}, J^{-}$and $J$ by $J^{ \pm}=2^{-1 / 2}( \pm D-X)$ and $J=J^{+} J^{-}=$ $2^{-1}\left(-D^{2}+X^{2}-I\right)$ (the Schrödinger representation of the one-dimensional harmonic oscillator) then it is well known $[8,13,23]$ that the $h_{n}$ are the eigenfunctions also of $J$, satisfying $J h_{n}=n h_{n}$. The infinitesimal generator of the Lie group $\left\{F_{\theta}\right\}$ is $-\mathrm{i} J$ $[16,22]$ so $F_{\theta}=e^{-\mathrm{i} \theta J}$. The set of operators $\left\{I, J, J^{ \pm}\right\}$constitutes a basis for an irreducible representation of a 4-dimensional Lie algebra and was used to develop the $F_{\theta}$-invariant uncertainty principles $[15,17]$ mentioned earlier.

The kernel of the integral transform $F_{\theta}$ is no longer a group character, so many of the elementary properties enjoyed by Fourier transforms no longer apply in their simple general shape.

The parity operator $F^{2}$ (mapping $f(t)$ to $f(-t)$ ) is just $F_{\pi}$ so as $\left\{F_{\theta}\right\}$ is Abelian one has $F_{\theta} F^{2}=F^{2} F_{\theta}$ so under $F_{\theta}$, as under $F$, even functions transform into even functions and odd transform into odd.

Complex conjugation is related to the inverse transform $F_{-\theta}$ in the usual way:

$$
\overline{F_{\theta} f}=F_{-\theta} \bar{f} \text {. }
$$

Defining the translation and modulation operators $T_{a}$ and $M_{a}$ by $\left(T_{a} f\right)(t)=f(t-a)$ and $\left(M_{a} f\right)(t)=e^{\mathrm{i} a t} f(t) \quad(a \in \mathbb{R})$ the standard Fourier transform results appear as $F T_{a}=M_{-a} F$ and $F M_{a}=T_{a} F$. From (8) one gets the corresponding results for $F_{\theta}$ :

$$
F_{\theta} T_{a}=\exp \left(-\frac{\mathrm{i}}{2} a^{2} \sin \theta \cos \theta\right) T_{a \cos \theta} M_{-a \sin \theta} F_{\theta}
$$




$$
F_{\theta} M_{a}=\exp \left(\frac{\mathrm{i}}{2} a^{2} \sin \theta \cos \theta\right) T_{a \sin \theta} M_{a \cos \theta} F_{\theta}
$$

or the combined result

$$
F_{\theta} T_{a} M_{b}=e^{\mathrm{i} \phi} T_{\alpha} M_{\beta} F_{\theta}
$$

where

$$
\phi=\phi(a, b, \theta)=\frac{1}{2} \sin \theta\left[\left(a^{2}-b^{2}\right) \cos \theta+2 a b \sin \theta\right]
$$

and

$$
\left[\begin{array}{l}
\alpha \\
\beta
\end{array}\right]=\left[\begin{array}{cc}
\cos \theta & \sin \theta \\
-\sin \theta & \cos \theta
\end{array}\right]\left[\begin{array}{l}
a \\
b
\end{array}\right]
$$

showing that a simple translation or modulation of the signal leads to both a translation and a modulation (together with a phase shift) of its fractional transform. From these one can see the corresponding effects on the energy density function $\left|F_{\theta} f\right|^{2}$ :

$$
\left|F_{\theta} T_{a} M_{b} f\right|^{2}=T_{a \cos \theta+b \sin \theta}\left|F_{\theta} f\right|^{2}
$$

\section{The Wigner distribution and the fractional Fourier transform}

The Wigner time-frequency distribution has been discussed recently along with others from the point of view of their membership of Cohen's generalized class of phase-space distributions and the constraints imposed on this class if the resulting distributions are to have certain desired properties, for example, [3, 2, 9]. For convenience in what follows write $f_{\theta}=F_{\theta} f$ (so $f_{0}=F_{0} f=f$ and $f_{\pi / 2}=F_{\pi / 2} f=F f$ ). To each kernel function $\Phi$ the corresponding Cohen representation $C_{f}(\mathbf{x}, \Phi)$ of the one-dimensional signal $f(f: \mathbb{B} \rightarrow \mathbb{C})$ on the two-dimensional $\mathbf{x}$-space, where $\mathbf{x}=(t, \omega) \in \mathbb{R}^{2}$, can be written:

$$
\begin{array}{r}
C_{f_{0}}(\mathbf{x}, \Phi)=(2 \pi)^{-3 / 2} \int_{\mathbb{R}^{3}} \exp [\mathrm{i}(\xi t-\tau \omega-\xi u)] \Phi(\xi, \tau ; \mathbf{x}) \\
f_{0}\left(u+\frac{\tau}{2}\right) \bar{f}_{0}\left(u-\frac{\tau}{2}\right) d u d \tau d \xi
\end{array}
$$

The particular case $\Phi=1$ yields the Wigner distribution $W_{f}$ :

$$
W_{f_{0}}(\mathbf{x})=C_{f_{0}}(\mathbf{x}, 1)=(2 \pi)^{-1 / 2} \int_{\mathbb{R}} e^{-\mathrm{i} \tau \omega} f_{0}\left(t+\frac{\tau}{2}\right) \bar{f}_{0}\left(t-\frac{\tau}{2}\right) d \tau
$$




$$
\text { (or, equivalently, } \left.W_{f_{0}}(\mathbf{x})=(2 \pi)^{-1 / 2} \int_{\mathbb{R}} e^{i \xi t} f_{\pi / 2}\left(\omega+\frac{\xi}{2}\right) \bar{f}_{\pi / 2}\left(\omega-\frac{\xi}{2}\right) d \xi\right) \text {. }
$$

If " $t$ " and " $\omega$ " are to be interpretable as time and frequency variables in $C_{f}$ then time and frequency translations of the signal $f$ must produce the corresponding translations in $C_{f}$; that is, defining translators $T_{\mathbf{a}_{\theta}}$ in the $\theta$ direction on functions $\Psi(\mathbf{x})$ by $T_{\mathbf{a}_{\theta}} \Psi(\mathbf{x})=\Psi\left(\mathbf{x}-\mathbf{a}_{\theta}\right)$ where $\mathbf{a}_{\theta}=a(\cos \theta, \sin \theta)$ then one must have

$$
C_{T_{a} f}=T_{\mathrm{a}_{0}} C_{f} \quad \text { and } \quad C_{M_{a} f}=T_{\mathrm{a}_{\pi / 2}} C_{f} .
$$

Recalling that $M_{a} f_{0}=F_{-\pi / 2} T_{a} f_{\pi / 2}$ the two requirements (3) generalize to the more stringent requirement to cover translations of all the fractional transforms $f_{\theta}$ along their axes:

$$
\forall \theta \in \mathbb{T} \quad C_{F_{-\theta} T_{a} f_{\theta}}=T_{\mathbf{a}_{\theta}} C_{f_{0}} .
$$

Properties (3) alone entail the independence of $\Phi$ from $\mathbf{x}$ (see [3]) and I assume this from now on. It is then clementary to show that this assumption is sufficient to satisfy (4) also.

For the marginal distribution of $C_{f}$ along the $t$-axis (the 0-direction) to represent the signal power and the marginal distribution along the $\omega$-axis (the $\pi / 2$-direction) to represent the spectral energy density, $C_{f}$ must satisfy

$$
(2 \pi)^{-1 / 2} \int_{\mathbb{R}} C_{f_{0}}(t, \omega) d \omega=\left|f_{0}(t)\right|^{2} \quad \text { and } \quad(2 \pi)^{-1 / 2} \int_{\mathbb{R}} C_{f_{0}}(t, \omega) d t=\left|f_{\pi / 2}(\omega)\right|^{2} .
$$

It is well known that the Wigner distribution (2) does satisfy the two requirements (5) as also do some other distributions of the Cohen class (1), for example (see [3]), those defined by taking either $\Phi(\xi, \tau)=e^{i a \xi \tau}$ or $\Phi(\xi, \tau)=\cos (a \xi \tau)$ (see [3]).

The two requirements (5) combine and naturally generalize to the more stringent requirement that

$$
\forall \theta \in \mathbb{T} \quad(2 \pi)^{-1 / 2} \int_{\ell(r, \theta)} C_{f}(\mathbf{x}) d \ell=\left|f_{\theta}(r)\right|^{2}
$$

where $d \ell$ is the element of Euclidean arc length along the line $\ell(r, \theta)$ whose equation is $x_{1} \cos \theta+x_{2} \sin \theta=r$. Equation (6) is precisely the statement that the energy density function $\left|f_{\theta}(r)\right|^{2}$ of the fractional Fourier transform $f_{\theta}$, now regarded as a function on $\mathbb{R}^{2}$ in polar coordinates $r$ and $\theta$, is the Radon transform of the phase-space distribution $C_{f}$ (see [6]).

THEOREM. There is exactly one member of the Cohen class of phase-space distributions that satisfies the generalized translation property (4) and the generalized marginal distribution (or Radon transform) property (6) and that is the Wigner distribution $W_{f}$. 
PROOF. I first show that the Wigner distribution satisfies (6). Define new variables $r, y$ in the $t-\omega$ plane along axes at angle $\theta$ to the $t, \omega$ axes by the substitution

$$
\left[\begin{array}{l}
t \\
\omega
\end{array}\right]=\left[\begin{array}{cc}
c & -s \\
s & c
\end{array}\right]\left[\begin{array}{l}
r \\
y
\end{array}\right] \quad(\text { where } c=\cos \theta \text { and } s=\sin \theta)
$$

and write $W_{\theta, f}(r, y)=W_{f}(t, \omega)$ then, using (2),

$$
\begin{aligned}
(2 \pi)^{-1 / 2} \int_{\ell(r, \theta)} W_{f}(\mathbf{x}) d \ell & =(2 \pi)^{-1 / 2} \int_{\mathbb{R}} W_{\theta, f}(r, y) d y \\
& =(2 \pi)^{-1} \int_{\mathbb{R}^{2}} e^{-\mathbf{i}(s r+c y) \tau} f(c r-s y+\tau / 2) \bar{f}(c r-s y-\tau / 2) d \tau d y .
\end{aligned}
$$

Now put $s r+c y=u$ to get

$$
\begin{aligned}
(2 \pi)^{-1 / 2} & \int_{\ell(r, \theta)} W_{f}(\mathbf{x}) d \ell \\
= & (2 \pi|c|)^{-1} \int_{\mathbb{R}^{2}} e^{-\mathrm{i} u \tau} f\left(\frac{r}{c}-\frac{s}{c} u+\frac{\tau}{2}\right) \bar{f}\left(\frac{r}{c}-\frac{s}{c} u-\frac{\tau}{2}\right) d \tau d u .
\end{aligned}
$$

The energy density $\left|f_{\theta}(r)\right|^{2}$ of the fractional transform $f_{\theta}$ is, using (8) of Section 2 ,

$$
\begin{aligned}
&\left|f_{\theta}(r)\right|^{2}=(2 \pi|s|)^{-1} {\left[\int_{\mathbb{R}} \exp \left\{-\frac{\mathrm{i}}{2 s}\left(-\left(w^{2}+r^{2}\right) c+2 w r\right)\right\} f(w) d w\right] \times } \\
& {\left[\int_{\mathbb{R}} \exp \left\{+\frac{\mathrm{i}}{2 s}\left(-\left(v^{2}+r^{2}\right) c+2 v r\right)\right\} \bar{f}(v) d v\right] } \\
&=(2 \pi|s|)^{-1} \int_{\mathbb{R}^{2}} \exp \left[-\frac{\mathrm{i}}{2 s}\left\{-\left(w^{2}-v^{2}\right) c+2(w-v) r\right\}\right] f(w) \bar{f}(v) d w d v
\end{aligned}
$$

Now put $w=(-s u+r) / c+\tau / 2$ and $v=(-s u+r) / c-\tau / 2$ to get

$$
\left|f_{\theta}(r)\right|^{2}=(2 \pi|c|)^{-1} \int_{\mathbb{R}^{2}} e^{-\mathrm{i} u \tau} f\left(\frac{r}{c}-\frac{s}{c} u+\frac{\tau}{2}\right) \bar{f}\left(\frac{r}{c}-\frac{s}{c} u-\frac{\tau}{2}\right) d \tau d u .
$$

Comparing (8) and (9) shows that $W_{f}$ satisfies (6); that is that the energy density $\left|f_{\theta}(r)\right|^{2}$ of the fractional Fourier transform is the Radon transform of the Wigner distribution.

To show that the Wigner distribution is the only function with this property one needs a uniqueness theorem for the Radon transform. As well as that in Radon's original 1917 paper (reproduced in facsimile in Helgason [12] and in English translation in Deans [6]) there are several uniqueness theorems relating to the Radon transform. Theorem 2.4 in Helgason [12], the "Schwartz theorem", simplified here for $\mathbb{R}^{2}$, is briefly as follows. 
Let $g$ be a function on $\mathbb{R}^{2}$; let $\ell(r, \mathbf{w})$ be the line in $\mathbb{R}^{2}$ specified by the unit vector $\mathbf{w}=\left(w_{1}, w_{2}\right)=(\cos \theta, \sin \theta)$ and the parameter $r \in \mathbb{R}$ through the equation $\mathbf{x} \cdot \mathbf{w}=r$ and let $\mathbb{P}^{2}$ denote the set of all lines in $\mathbb{B}^{2}$. Then the Radon transform $\mathscr{R}$ maps a function $g$ on $\mathbb{R}^{2}$ to a function $\mathscr{R} g$ on $\mathbb{P}^{2}$ according to the definition (omitting the constant $\mathrm{I}$ include in (6))

$$
\mathscr{R} g(\ell(r, \mathbf{w}))=\int_{\mathbf{x} \in \ell} g(\mathbf{x}) d m(\mathbf{x})
$$

where $d m(\mathbf{x})$ is the Euclidean measure on $\ell$ so that is, writing $\mathbf{w}^{\perp}=(-\sin \theta, \cos \theta)$,

$$
\mathscr{R} g(\ell(r, \mathbf{w}))=\int_{\mathbb{R}} g\left(r \mathbf{w}+s \mathbf{w}^{\perp}\right) d s .
$$

Let $\mathscr{S}\left(\mathbb{R}^{2}\right)$ denote the Schwartz space of rapidly decreasing $C^{\infty}$ functions on $\mathbb{R}^{2}$ and let $\mathscr{S}_{H}\left(\mathbb{P}^{2}\right)$ denote the space of all those $C^{\infty}$ functions $\varphi$ on $\mathbb{P}^{2}$ such that $\forall k \in \mathbb{N} \int_{\mathbb{R}} \varphi(\ell(r, \mathbf{w})) r^{k} d r$ is a homogeneous polynomial of degree $k$ in $w_{1}$ and $w_{2}$. Then $\mathscr{R}$ is a bijection from $\mathscr{S}\left(\mathbb{R}^{2}\right)$ to $\mathscr{S}_{H}\left(\mathbb{P}^{2}\right)$.

Now because $\mathscr{S}\left(\mathbb{R}^{2}\right)$ is dense in $L^{2}\left(\mathbb{R}^{2}\right) \mathscr{R}$ is a bijection in the $L^{2}$ sense from $L^{2}\left(\mathbb{R}^{2}\right)$ and therefore if the equation $\left|f_{\theta}(r)\right|^{2}=\mathscr{R} C_{f}(\ell(r, \theta))$ is satisfied for $C_{f}=W_{f}$, as I have just shown, it is satisfied by no function distinct from $W_{f}$ in the $L^{2}$ sense. Therefore although perhaps other candidates from the Cohen class share the limited property (5) and even some other desirable properties [3] only the Wigner distribution has the Radon transform property (6), corresponding to the natural imbedding of the property of yielding two correct marginal distributions $|f|^{2}$ and $|F f|^{2}$ into that of yielding all the correct marginal distributions, the fractional Fourier transform energy densities $\left|F_{\theta} f\right|^{2}$.

There is an inversion formula involving an integral of $W_{f}$ along a line in the $\pi / 2$-direction that gives $f(t)$ except for a phase constant [24]:

$$
f(t) \bar{f}(0)=(2 \pi)^{-1 / 2} \int_{\mathbb{R}} W_{f}(t / 2, \omega) e^{\mathrm{i} t \omega} d \omega
$$

and a similar one involving an integral of $W_{f}(t, \omega / 2)$ in the $\pi$-direction that gives $\hat{f}(\omega) \overline{\hat{f}}(0)$. In the light of the theorem just proved one expects the following proposition to be true.

PROPOSITION. In the notation of the theorem and its proof an integral corresponding to (10) involving $W_{\theta, f}(r / 2, y)$ along the line $\ell(r, \theta)$ yields the fractional Fourier transform $f_{\theta}(r)$ to within a phase constant $\bar{f}_{\theta}(0)$ :

$$
f_{\theta}(r) \bar{f}_{\theta}(0)=(2 \pi)^{-1 / 2} \int_{\mathbb{R}} W_{\theta, f}(r / 2, y) e^{i r y} d y .
$$


PROOF. Write $S_{\theta, f}(r)$ for the right-hand side of (11); substitute the appropriate expression for $W_{\theta, f}$ using (7); write $S_{\theta, f}$ as a double integral and then

$$
\begin{aligned}
S_{\theta . f}(r)=(2 \pi)^{-1} \int_{\mathbb{R}^{2}} & \exp [-\mathrm{i}\{(s r / 2+c y) p-r y\}] \\
& f(c r / 2-s y+p / 2) \bar{f}(c r / 2-s y-p / 2) d p d y .
\end{aligned}
$$

Change the variable $y$ by putting $u=c r / 2-s y-p / 2$ so then (invoking Fubini)

$$
\begin{gathered}
S_{\theta, f}(r)=(2 \pi|s|)^{-1} \int_{\mathbb{R}} \int_{\mathbb{R}} \exp \left[\frac{-\mathrm{i}}{2 s}\left\{-\left(r^{2}+p^{2}\right) c+2 r p-2 u(c p-r)\right\}\right] \\
f(u+p) d p \bar{f}(u) d u .
\end{gathered}
$$

Replace $p$ by $p-u$ in the inner integral to get

$$
S_{\theta, f}(r)=(2 \pi|s|)^{-1} \int_{\mathbb{R}} \int_{\mathbb{R}} \exp \left[\frac{-\mathrm{i}}{2 s}\left\{-\left(r^{2}+p^{2}\right) c+2 r p+u^{2} c\right\}\right] f(p) d p \bar{f}(u) d u
$$

which one can now write as a product of integrals and confirm from (8) of Section 2 that it is equal to $f_{\theta}(r) \bar{f}_{\theta}(0)$.

Obviously one can construct the Wigner distribution from any fractional Fourier transform $f_{\theta}$ by applying the defining formula (2a) to $f_{\theta}$ and then rotating the result.

\section{Conclusion}

From the results here one can see that the Wigner distribution is a specially distinguished member of the Cohen class of phase-space distributions and that (except for phase constants) there is a complete correspondence determined by the Radon transform between $\left\{f_{\theta}(r)\right\}_{\theta \in \mathbb{\pi}}$, the set of all the Condon-Bargmann fractional Fourier transforms of a function $f$, and the Wigner distribution, $W_{f}(\mathbf{x})$, of the function. Each is an invertible integral transform of $f$ that gives a time-frequency or intermediate representation of a signal. The exponential expression appearing in the definition of the Wigner distribution is conveniently bilinear but the transform itself is not a linear transform. The fractional Fourier transform, on the other hand, has the inconvenient quadratic expression in its exponential but it is a linear transform and this may recommend its application in some studies. Defining scaling and "chirping" operators $S_{a}(a \in \mathbb{R}-\{0\})$ and $C_{b}(b \in \mathbb{R})$ by $S_{a} f(t)=|a|^{1 / 2} f(a t)$ and $C_{b} f(t)=\exp \left(-\mathrm{i} b t^{2} / 2\right) f(t)$ then one can always write the fractional Fourier transform operator as a product of these with the usual Fourier operator $F$ : 


$$
F_{\theta}=a_{\theta} C_{\cot \theta} S_{\csc \theta} F C_{\cot \theta}
$$

where $a_{\theta}=\exp [(-i / 2)\{(\pi / 2) \operatorname{sgn} \theta-\theta\}]$, a phase constant. This representation might be a starting point for an adaptation of the fast Fourier transform algorithm for numerical approximations to $f_{\theta}$.

\section{References}

[1] V. Bargmann, "On a Hilbert space of analytic functions and an associated integral transform. Part I.", Comm. Pure Appl. Math. 14 (1961) 187-214.

[2] B. Bouachache, B. Escudie, P. Flandrin and J. Gréa, "Sur une condition nécessaire et suffisante de positivité de la représentation conjointe en temps et fréquence des signaux d'énergie finie", $C . R$. Acad. Sc. Paris serie A 288 (1979) 307-309.

[3] T. A. C. M. Claasen and W. F. G. Mecklenbräuker, "The Wigner distribution - a tool for timefrequency signal analysis III", Philips J. Res. 35 (1980) 372-389.

[4] L. Cohen, "Generalized phase-space distribution functions", J. of Math. Phys. 7 (1966) 781-786.

[5] E. U. Condon, "Immersion of the Fourier transform in a continuous group of functional transformations", Proc. Nat. Acad. Sci. USA 23 (1937) 158-164.

[6] S. R. Deans, The Radon Transform and Some of its Applications (Wiley, New York, 1983).

[7] H. Dym and H. P. McKean, Fourier Series and Integrals (Academic Press, New York, 1972).

[8] A Erdélyi, W. Magnus, F. Oberhettinger and F. G. Tricomi, Higher Transcendental Functions, Vol. II (McGraw Hill, New York, 1953).

[9] P. Flandrin and B. Escudié, "Time and frequency representation of finite-energy signals : a physical property as a result of an Hilbertian condition", Signal Processing 2 (1980) 93-100.

[10] V. Fock, "Verallgemeinerung und Lösung der Diracschen statistischen Gleichung", Z. Physik 49 (1928) 339-357.

[11] G. B. Folland, Harmonic analysis in phase space, Annals of Mathematics Studies (Princeton Univ. Press, Princeton, 1989).

[12] S. Helgason, The Radon transform, Progress in Mathematics Vol. 5 (Birkhaüser, Basel, 1980).

[13] W. Miller Jr., Lie Theory and Special Functions (Academic Press, New York, 1968).

[14] D. Mustard, "The fractional Fourier transform and the Wigner distribution", Applied Mathematics Preprint AM89/6 School of Mathematics, UNSW (1989).

[15] D. Mustard, "Uncertainty principles invariant under the fractional Fourier transform", J. Austral. Math. Soc. Ser. B 33 (1991) 180-191.

[16] D. Mustard, "Lie group imbeddings of the Fourier transform", Applied Mathematics Preprint AM87/13 School of Mathematics, UNSW (1987).

[17] D. Mustard, "Lie group imbeddings of the Fourier transform and a new family of uncertainty principles", in Proceedings of the Centre for Mathematical Analysis, Australian National University, Volume 16, Miniconferences on Harmonic Analysis and Operator Algebras (Canberra: 5-8 August and 2-3 December 1987) (1987) 211-222.

[18] H. M. Ozaktas, B. Barshan, D. Mendlovic and L. Onural, "Convolution, filtering, and multiplexing in fractional Fourier domains and their relation to chirp and wavelet transforms", J. Opt. Soc. Am. A 11 (1994) 547-559.

[19] Y. Nievergelt, SIAM Review 28 (1986) 79-84. 
[20] A. Papoulis, Signal Analysis (McGraw-Hill, New York, 1977).

[21] Proc. IEEE Special Issue on Computerized Tomography (March 1983).

[22] M. Taylor, "Noncommutative microlocal analysis, Pt. I", Memoirs of the AMS 52 (1984), No. 313.

[23] N. Ja. Vilenkin, Special Functions and Theory of Group Representations (Izd. Nauka., Moscow, 1965 (in Russian)) Special Functions and the Theory of Group Representations (AMS Transl. Vol.22, Providence, R.I., 1968 (English Transl.)).

[24] E. Wigner, "On the quantum correction for thermodynamic equilibrium", Phys. Rev. 40 (1932) 749-759. 